\title{
Erratum: Dynamical structure factor of the triangular-lattice antiferromagnet [Phys. Rev. B 88, 094407 (2013)]
}

\author{
M. Mourigal, W. T. Fuhrman, A. L. Chernyshev, and M. E. Zhitomirsky
}

(Received 19 February 2016; published 7 March 2016)

DOI: 10.1103/PhysRevB.93.099901

Figure 4(a) of our paper contains a mistake traced to a typo in the code combining different components of the dynamical structure factor. As a result, the total dynamical structure factor $S^{\text {tot }}(\mathbf{q}, \omega)$, Eq. (5), for $S=1 / 2$ along the $K \Gamma$ segment of Fig. $4($ a) was not entirely correct. While the overall differences of the corrected plot given below with the published figure are minor, the corrected version of Fig. 4 clearly shows a fainter contribution to the structure factor at lower energies. This brings Fig. 4 in accord with our own Fig. 3(c) of the same paper and with the upper-left panel of Fig. 4 in Supplemental Material of Ref. [1]. None of the other figures or results of our paper are affected.

We acknowledge a discussion with Pavel Maksimov, which has led to this finding.

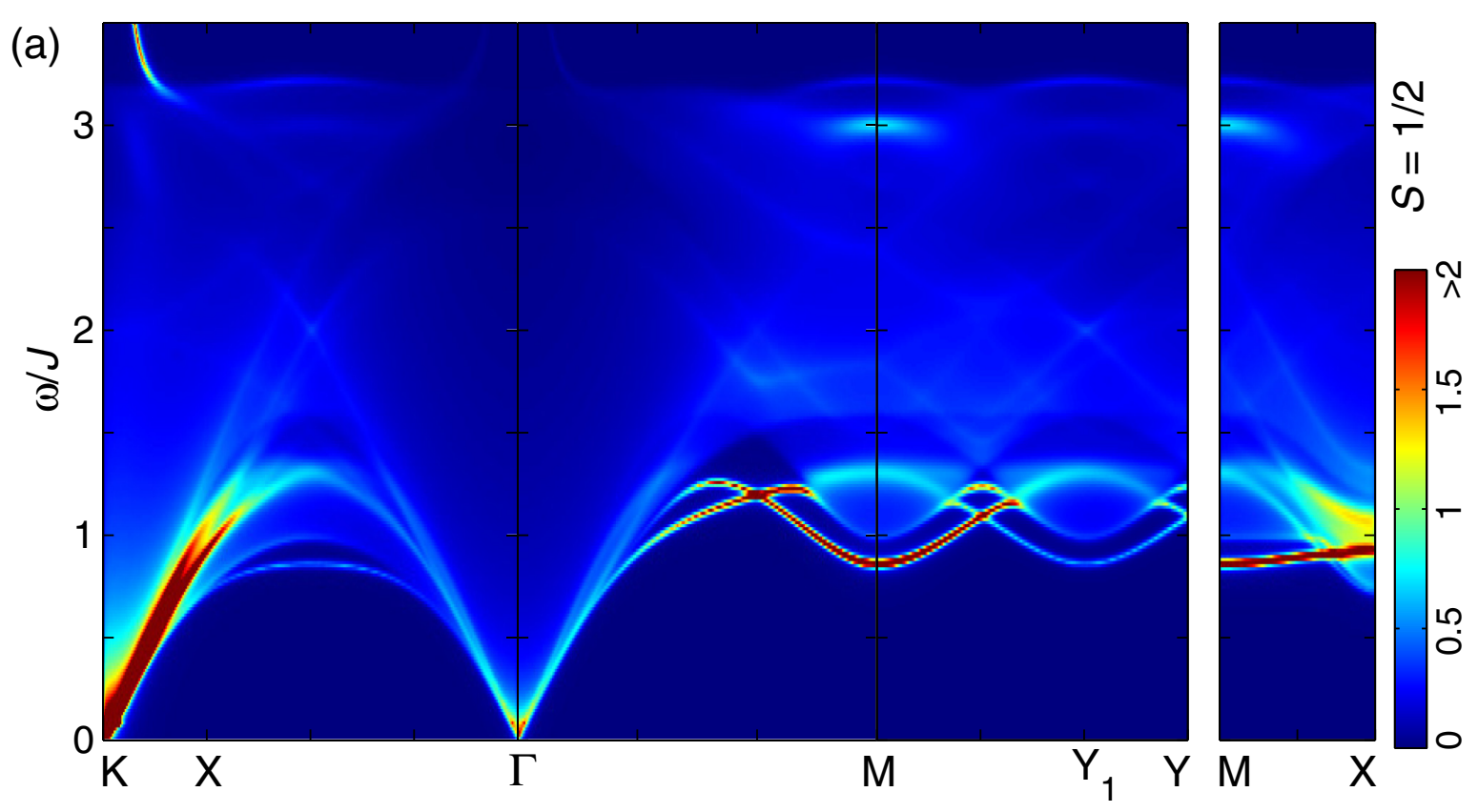

FIG. 4. Intensity plots of the momentum and energy dependence of the total dynamic structure factor $S^{\text {tot }}(\mathbf{q}, \omega)(5)$ for $S=1 / 2$ along the high-symmetry paths in the Brillouin zone shown in Fig. 1(b).

[1] J. Ma, Y. Kamiya, Tao Hong, H. B. Cao, G. Ehlers, W. Tian, C. D. Batista, Z. L. Dun, H. D. Zhou, and M. Matsuda, Phys. Rev. Lett. 116, 087201 (2016). 